dopaminergic medication. The second objective was to explore the relationship between frequency of SoP experiences and dopaminergic drug, drug dosage and length of drug exposure. The experimental hypothesis was that SoP symptoms in PD would present more frequently in patients treated longer and with higher dopaminergic drug doses.

Background. PD is a debilitating neurodegenerative disorder. Psychiatric symptoms are common and associated with impaired quality of life and higher treatment costs. PD psychosis often starts with 'minor hallucinations', the most common being a false 'sense of presence' (SoP), the vivid sensation that someone else is nearby when nobody is there. SoP symptoms typically do not cause significant distress but may act as a prognostic marker for future severe psychosis and may prompt alteration of treatment or reduction in dopaminergic drug dosage. This study aimed to extend prior research by characterizing SoP further and investigating the link with dopaminergic medication.

Method. This was a retrospective, cross-sectional study. Twenty-one patients diagnosed with PD completed a questionnaire to identify presence of SoP symptoms, duration of symptoms, timing of onset related to dopaminergic treatment and the frequency of symptoms in relation to current levodopa equivalent dose (LED). Descriptive frequencies were compared using a two-tailed t-test. Multiple regression analysis was conducted to assess the relationship between frequency of SP experiences, levodopa equivalent dose and length of drug exposure.

Result. Sixteen of twenty-one patients reported experiencing SoP symptoms. Patients who had not experienced SoP symptoms had a significantly lower LED than those who had experienced these symptoms. There were no other significant differences between the groups. No statistical significance was shown on regression analysis; however our study was not adequately powered for the regression analysis as the number of participants was too low.

Conclusion. This study confirms that SoP symptoms are common among patients with PD and supports a correlation between the total daily equivalent dose of levodopa and SoP symptoms. It does not provide evidence for a temporal relationship between onset of SoP symptoms and duration of dopaminergic treatment. The study was insufficiently powered and a larger study is required to investigate further.

\section{A two-year mirror-image study of the effect of treatment with paliperidone and aripiprazole long-acting injections on need for inpatient care and home treatment intervention

\author{
Shay-Anne Pantall ${ }^{1 *}$, Joseph Pilsbury ${ }^{2}$, Le Gan ${ }^{2}$ \\ and Lisa Brownell ${ }^{1}$ \\ ${ }^{1}$ Birmingham and Solihull Mental Health NHS Foundation Trust and \\ ${ }^{2}$ University of Birmingham \\ ${ }^{*}$ Corresponding author.
}

doi: 10.1192/bjo.2021.745

Aims. To evaluate the effect of the use of aripiprazole and paliperidone long acting injections on healthcare resource use

Background. Long acting injections of second-generation antipsychotics such as paliperidone and aripiprazole have become more commonly prescribed over the past decade. They have much higher acquisition costs when compared to first generation depot antipsychotics. It is therefore essential to demonstrate their tolerability and cost-effectiveness.

Method. We undertook an observational, retrospective two-year mirror study for all patients who started treatment with paliperidone long acting injection between January and June $2016(\mathrm{n}=47)$ or aripiprazole long acting injection between April 2014 and July $2017(\mathrm{n}=93)$. Clinical notes were examined to determine the number of admissions, inpatient days, home treatment episodes and number of home treatment days, in the 12 months preceding and following the commencement of the long acting injection.

Result. 70\% remained on paliperidone and $62 \%$ remained on aripiprazole at the end of the one-year period.

There was a significant reduction in occupied bed days in those treated with paliperidone from 78.2 days in the year before this treatment was started to 25.4 days in the year after $(p=0.002)$. There was a significant reduction in occupied bed days in those treated with aripiprazole from 66.51 days to 32.7 days $(p=0.0006)$.

There was no significant reduction in days spent under the care of home treatment teams for individuals treated with either of these medicines.

Conclusion. Treatment with either paliperidone or aripiprazole long-acting injection was associated with a reduction in admissions and occupied bed days of a magnitude that delivered an overall cost-saving despite the high drug acquisition costs. It remains to be determined how these reductions compare with other second-generation long-acting injections and firstgeneration depot antipsychotics.

\section{Using qualitative methods, what factors do child \&} adolescent higher trainees and new consultants (within 1 year of appointment), report in choosing their higher training subspecialty?

\section{Mary Parker*, Jim Boylan and Nicholas Wolstenholme}

Tees, Esk \& Wear Valley NHS Foundation Trust

${ }^{\star}$ Corresponding author.

doi: 10.1192/bjo.2021.746

Aims. This Research aims to identify factors reported by recent and current trainees in choosing Child \& Adolescent (C\&A) Psychiatry for their higher training/career path. The hypothesis is that via thematic analysis prominent factors will emerge to inform future recruitment and retention.

Background. The need to attract more doctors into Psychiatry has been identified by NHS, Royal College of Psychiatrists and the Media; Health Education England figures show core psychiatry had lowest fill rates of any specialty in $2016,17 \& 18$.

Some subspecialties experience particular difficulties e.g. C\&A. Royal College of Psychiatry analysis of workforce in March 2018 showed the numbers of C\&A psychiatrists of all grades have fallen by $6.3 \%$ in four years and for consultants alone the decrease was $6.9 \%$ over the same period.

However, very little research has been completed investigating why trainees might choose C\&A Psychiatry with a literature search revealing only one report of C\&A trainees views in the UK in 2006.

Method. A qualitative design was chosen to provide insight into the factors affecting participants in choosing their career.

The theoretical framework supporting the study relates to capturing experience via a case study approach, aiming to explore reported issues in a real life context and considering similarities in the cases to inform future recruitment.

The Research was approved by The Health Research Authority and local Research \& Development departments.

Purposeful sampling was used with voluntary participation following informed consent and non-identifiable demographic data were collected and analysed quantatively.

Semi-structured interviews to saturation were conducted with fourteen $(\mathrm{N}=14)$ participants asked questions exploring their 